\title{
Analysis and Assessment of Knowledge Sharing Risk in the Virtual Enterprise
}

\author{
Tianhui You, Zhu Zhu, Zhuchao Yu \\ School of Business Administration, Northeastern University, Shenyang, P.R. China, 110004
}

\begin{abstract}
A method to analyze and assess knowledge sharing risks in the virtual enterprise is proposed. Firstly, based on risks analysis, an index system is set up to assess such risks as the core competence losing risk, the enterprise culture risk, the knowledge spillover effect risk and the moral risk, etc.. Then, according to the index system and the characteristics of linguistic assessment information provided by experts, a multiindex decision-making method based on the fuzzy linguistic assessment information is proposed using LWD operator and LOWA operator developed in recent years. Finally, an example is given to explain the method proposed.
\end{abstract}

Keywords: Virtual enterprise, Knowledge sharing, Risk, LWD operator, LOWA operator

\section{Introduction}

Along with society's development and the technical progress, market competition is becoming global and intensive, and the business environment is changing quickly and greatly. It is a great challenge to the traditional management and administration, and calls for revolution. Modern technologies such as computer, information, network and communication technologies as well as artificial intelligence provide powerful support for the implementation of management stratagem in the virtual enterprise [1]. The virtual enterprise is a temporary alliance of some mutually independent service process or the enterprises to share skills, core competencies, resources and risk in order to respond to business opportunities. As the inherent market characteristic and the production characteristic in the knowledge economy time, the knowledge becomes the most important resources in the enterprise production process, and also is "the only resources to provide the competitive advantage”. From the knowledge-based view of the enterprise theory, the knowledge activity passes through the total life cycle in the virtual enterprise, the cooperation process of the virtual enterprise (in particular knowledge alliance virtual enterprise) is the knowledge sharing process in fact. The virtual enterprise obtains $1+1>2$ the knowledge coordination multiplies effect through sharing different enterprises' appropriation knowledge. But, there are uncertainty caused by some mentality motive factors in knowledge sharing, which may cause some risks in knowledge sharing. At present, the researches on knowledge sharing and knowledge management in the virtual enterprise have already aroused the attention from scholars. The researches on knowledge sharing focuses include such aspects as object [2], means [3, 4], subject [5, 6] and economy [7, 8] of knowledge sharing. Some scholars research on knowledge management in the virtual enterprises [9, 10], But, the researches on knowledge sharing risks in the virtual enterprise are still rare [11]. So, our research addresses this gap by studying the risks of knowledge sharing in virtual enterprise, and provides a multi-index decision-making method to evaluate the risks.

\section{The Risk Index System}

In the virtual enterprise, knowledge sharing can be divided into two kinds of situations, namely knowledge sharing carries on in the member enterprise boundary and carries on between the member enterprises. Because the former equates with the common enterprise's knowledge sharing question, therefore, the research on knowledge sharing risks in the virtual enterprise mainly aims at the latter one. The virtual enterprise can realize different organization's knowledge sharing cross the space and time and cultural, its knowledge distribution is broader than the traditional enterprise's. As a result of this kind of knowledge different distribution, the correlative information incompletion and the knowledge innovation process uncertainty, this causes knowledge sharing more difficult between members in the virtual enterprise, along with which the risk is also much bigger. The main knowledge sharing risks in the virtual enterprise are found out through analyzing knowledge sharing process, which mainly are the core competence losing risk, the enterprise culture risk, the knowledge spillover effect risk and the moral risk.

\subsection{The core competence losing risk}


The goal of knowledge sharing in the virtual enterprise is to conform and integrate knowledge through each kind of foundation or the fully and widespread sharing of the specialized knowledge, and carries on the innovation and the fast product innovation in this foundation. The virtual enterprise knowledge sharing mainly is the member enterprises complementary knowledge sharing, the knowledge sharing scope and the sharing degree should be given in the contract to stipulate. During the knowledge sharing process, if the knowledge source side does not pay great attention to protect their own core knowledge or the knowledge accepters use conditions such as imperfect contract and unsymmetrical information, and exercise the opportunism to absorb knowledge from the partner excessively, the core competence losing risk will be induced.

\subsection{The enterprise culture risk}

The enterprise culture refers to staff's value, faith and behavior in common in an enterprise. The core of enterprise culture is the enterprise value, and which is embodied by the enterprise system that regularizes the staff's behavior. Because the members in the virtual enterprise come from different enterprises, each member enterprise all has its own unique enterprise culture, if a virtual enterprise has not formed new values that regards sharing and collaboration as core content, the staff will lack of the acceptance to knowledge sharing. They may think that their advantages in the enterprise come from certain knowledge they know and others do not know, so refuse to share their own knowledge with others out of the protection of the individual interests. System absence and system invalidation will increase the system cost of knowledge sharing, thus cause the knowledge sharing risk in a virtual enterprise.

\subsection{The knowledge spillover effect risk}

Any main body have to pay to obtain the knowledge, therefore the knowledge acquisition process may be regarded as one kind of investment, but the knowledge accumulation can form somebody unique cognition ability as well as to some thing present situation or the development tendency judgment ability, other people gained this cognition or the judgment by some way, namely called the knowledge spillover. The knowledge spillover also is a knowledge diffuse way, which is passive, unconsciousness, non-voluntary, divulged, or performances as a technical trade in information holding. The knowledge spillover effect is refers to the receiver or the demander to digest and absorb knowledge and then causes the knowledge innovation going with other economical growth influence etc.. In the virtual enterprise, the member enterprise may lose the competitive advantage which is imitated by the existence or potential competition rival due to the knowledge spillover effect, thus leads to the knowledge spillover effect risk.

\subsection{The moral risk}

Before the virtual enterprise being set up, various member enterprises can constitute a contract to restrict the scope and the degree of sharing knowledge. However, as a result of knowledge fuzziness, this kind of contract has the enormous limitation, which has left behind quite a few spaces for the opportunism behavior; each partner in the virtual enterprise partially has the relationship of principal-agent with the work team being sent out. Whether the agent faithfully carries out the principal's purpose or not, this also can result in the knowledge sharing moral risk.

\section{Assessment Method}

In order to evaluate the risks of knowledge sharing in the virtual enterprise, let $\boldsymbol{P}=\left\{P_{1}, P_{2}, \ldots, P_{q}\right\}(q \geq 2)$ be a non-empty and finite set of risk assessing indexes, where $P_{j}$ is the $j$ th risk index. Let $\boldsymbol{E}=\left\{E_{1}, E_{2}, \ldots\right.$, $\left.E_{m}\right\}(m \geq 2)$ be a non-empty and finite set of experts, where $E_{k}$ is the $k$ th expert. Let $\boldsymbol{R}^{k}=\left(r_{1}^{k}, r_{2}^{k}, \ldots\right.$, $\left.r_{q}^{k}\right)^{\mathrm{T}}$ be the weight vector of indexes given by $E_{k}$, where $r_{j}^{k}$ is chosen from a nature linguistic assessment terms set $\boldsymbol{L}$. $\boldsymbol{L}$ is a pre-established ordinal nature linguistic assessment term set consisting of odd linguistic terms, which describes the importance of $P_{j}$. Finally, let $\boldsymbol{A}=\left(a_{j}^{k}\right)_{m \times q}$ be an assessment matrix, where $a_{j}^{k}$ is the $k$ th expert's assessment value of the $j$ th index from a nature linguistic assessment terms set $\boldsymbol{S}$, a set just like $\boldsymbol{L}$. In this paper, a set of five terms $\boldsymbol{S}$ and $\boldsymbol{L}$ could be given such as $\boldsymbol{L}=\boldsymbol{S}=\left(S_{i}\right)=\left\{S_{0}=\operatorname{VL}(\right.$ Very Low $), S_{1}=\mathrm{L}(\mathrm{Low}), S_{2}=$ M(Moderate), $S_{3}=\mathrm{H}\left(\right.$ High), $S_{4}=\mathrm{VH}$ (Very High) $\}$.

Usually, in these cases, it is required that in the linguistic terms set there exists:

(1) A negation operator: $\operatorname{Neg}\left(S_{i}\right)=S_{j}$ such that $j=\mathrm{T}-i$ ( $\mathrm{T}+1$ is the cardinality).

(2) $S_{i} \leq S_{j} \Leftrightarrow i \leq j$. Therefore, there exists a $\min$ and a max operator.

In order to manage and calculate the linguist terms conveniently, the paper will propose a multi- 
index decision-making method to assess the risk of knowledge sharing in the virtual enterprise in virtue of the LWD (Linguistic Weighted Disjunction) operator [12] and LOWA (Linguistic Ordered Weighted Averaging) operator $[13,14]$. The calculation steps of the method are given as follows:

Step 1. Aggregate the risk indexes of linguist assessment information given by each expert. First, the linguist assessment information of indexes $a_{j}^{k}$ and weights $r_{j}^{k}$ given by each expert are aggregated into integrated values by virtue of LWD operator and LOWA operator using the formulation as follow:

$$
\left(a^{k}, r^{k}\right)=\phi\left[\left(a_{1}^{k}, r_{1}^{k}\right),\left(a_{2}^{k}, r_{2}^{k}\right), \cdots,\left(a_{q}^{k}, r_{q}^{k}\right)\right]
$$

where $k=1,2, \cdots, m ; a^{k}$ is the integrated value of knowledge sharing risk given by the expert $E_{k} ; r^{k}$ is the importance of the expert $E_{k}$ and $\phi$ is LWD operator. $a^{k}$ and $r^{k}$ can be calculated as follows:

$$
\begin{aligned}
& a^{k}=\max _{j=1,2, \cdots, q} \min \left(a_{j}^{k}, r_{j}^{k}\right), \quad k=1,2, \cdots, m \\
& r^{k}=\phi_{Q}\left(r_{1}^{k}, r_{2}^{k}, \cdots, r_{q}^{k}\right), \quad k=1,2, \cdots, m
\end{aligned}
$$

where $\phi_{Q}$ is LOWA operator. $\phi_{Q}$ is defined as:

$$
\begin{aligned}
\phi_{Q}\left(r_{1}^{k},\right. & \left.r_{2}^{k}, \cdots, r_{q}^{k}\right)=W^{\mathrm{T}} B \\
& =\xi^{q}\left\{w_{t}, r_{\sigma(t)}^{k}, t=1,2, \cdots, q\right\} \\
& =w_{1} \otimes r_{\sigma(1)}^{k} \oplus \xi^{q-1}\left\{\beta_{h}, r_{\sigma(h)}^{k}, h=2,3, \cdots, q\right\}
\end{aligned}
$$

where $\boldsymbol{W}=\left(w_{1}, w_{2}, \cdots, w_{q}\right)^{\mathrm{T}}$ is a weight vector, $w_{t} \in[0,1], \sum_{t=1}^{q} w_{t}=1, \beta_{h}=w_{h} / \sum_{t=2}^{q} w_{t}, h=2,3, \cdots q$ and $\boldsymbol{B}=\left\{b_{1}, b_{2}, \cdots b_{m}\right\}$ is a vector associated to $R^{k}$, $\boldsymbol{B}=\sigma(A)=\left(r_{s(1)}^{k}, r_{s(2)}^{k}, \cdots, r_{s(q)}^{k}\right)^{\mathrm{T}}$, where $r_{s(j)}^{k} \leq r_{s(i)}^{k}$, $\forall i \leq j$, with $\sigma$ being a permutation over the set of labels $R^{k} . \xi^{q}$ is the convex combination operator of $q$ terms. $\otimes$ is the general product of a term by a positive real number and $\oplus$ is the general addition of terms. If $q=2$, then $\xi^{q}$ is defined as:

$$
\begin{gathered}
\xi^{2}\left\{w_{t}, r_{\sigma(t)}^{k}, t=1,2\right\}=w_{1} \otimes S_{j} \oplus\left(1-w_{1}\right) \otimes S_{i}=S_{l} \\
S_{j}, S_{i} \in S(j \geq i)
\end{gathered}
$$

where $l=\min \left(\mathrm{T}, i+\operatorname{round}\left(w_{1} \cdot(j-i)\right)\right)$, “ $\operatorname{round}(\cdot)$ ” is the usual round operator, and $r_{\sigma(1)}^{k}=S_{j}, r_{\sigma(2)}^{k}=S_{i}$.
In the case of a non-decreasing proportional quantifier, $w_{t}$ is given by this expression

$$
w_{t}=Q(t / q)-Q((t-1) / q), \quad t=1,2, \cdots, q
$$

where $Q(u)$ is fuzzy quantifier operator, which is given by this expression:

$$
Q(u)=\left\{\begin{aligned}
0, & u<d \\
\frac{u-d}{f-d}, & d \leq u \leq f \\
1, & u>f
\end{aligned}\right.
$$

where $d, f, u \in[0,1]$. The corresponding parameter $(u, f)$ is $(0,0.5),(0.3,0.5)$ and $(0.5,1)$, respectively representing "half at least”, “majority”, "as much as possible” [12].

Step 2. Aggregate the integrated assessment values of each expert into the integrated assessment value of the expert group. $a^{k}$ and $r^{k}$ are integrated into the integrated assessment value of the expert group by virtue of LWD operator and LOWA operator, namely,

$$
(a, r)=\phi\left[\left(a^{1}, r^{1}\right),\left(a^{2}, r^{2}\right), \cdots,\left(a^{m}, r^{m}\right)\right]
$$

where $a$ is the assessment value of the expert group, $a \in S ; r$ is the credibility degree of the information given by the expert group, $r \in S . a$ and $r$ are calculated separately as follows:

$$
\begin{aligned}
& a=\max _{k=1,2, \cdots m} \min \left(a^{k}, r^{k}\right) \\
& r=\phi_{Q}\left(r^{1}, r^{2}, \cdots r^{m}\right)
\end{aligned}
$$

where the method to calculate $r$ is as same as the method given before to calculate $r^{k}$ and no longer go into details here.

Step 3. Judge the current situation of knowledge sharing risk in the virtual enterprise. The current situation of knowledge sharing risk in the virtual enterprise can be known by virtue of the value of $a$ calculated before, and meanwhile, the credibility degree of the information given by the expert group can be known by virtue of the value of $r$.

\section{Illustrative Example}

Let us suppose a virtual enterprise, which has four risk factors as core competence losing risk ( $\left.P_{1}\right)$, enterprise culture risk $\left(P_{2}\right)$, knowledge spillover effect risk $\left(P_{3}\right)$ 
and moral risk $\left(P_{4}\right)$. The virtual enterprise invites three experts (i.e., $E_{1}, E_{2}, E_{3}$ ) to assess the risk of knowledge sharing. The weight vectors and assessment matrix provided by experts are

$$
\begin{aligned}
\boldsymbol{R}^{1} & =(\mathrm{H}, \mathrm{M}, \mathrm{L}, \mathrm{H})^{\mathrm{T}}, \\
\boldsymbol{R}^{2} & =(\mathrm{VH}, \mathrm{H}, \mathrm{VL}, \mathrm{M})^{\mathrm{T}}, \\
\boldsymbol{R}^{3} & =(\mathrm{M}, \mathrm{M}, \mathrm{L}, \mathrm{M})^{\mathrm{T}} . \\
\boldsymbol{A} & =\left[\begin{array}{cccc}
\text { VH } & \text { VL } & \text { M } & \mathrm{M} \\
\mathrm{H} & \mathrm{L} & \mathrm{M} & \mathrm{L} \\
\mathrm{H} & \mathrm{VH} & \mathrm{VL} & \mathrm{H}
\end{array}\right]
\end{aligned}
$$

According to the formulas of (1) and (2), we can obtain $a^{1}=\mathrm{H}, a^{2}=\mathrm{H}$ and $a^{3}=\mathrm{M}$. We use the principle of "as much as possible", so the parameter $(u, f)$ corresponding to $Q(u)$ is $(0.5,1)$. According to the formulas of $(3) \sim(10)$, we can obtain $r^{1}=\mathrm{L}$, $r^{2}=\mathrm{L}, r^{3}=\mathrm{M}$. Then we obtain the assessment value of the expert group, namely, $a=\mathrm{M}$. Meanwhile, the credibility degree of the information given by the expert group is obtained, namely, $r=\mathrm{L}$. Finally, we know that the risk assessment result of knowledge sharing in this virtual enterprise is "Moderate".

\section{Conclusions}

This paper analyzes the risks of knowledge sharing in the virtual enterprise and proposes a multi-index decision-making method to assess these risks. The method is based on the linguistic assessment information using LWD operator and LOWA operator. And the credibility degree of the information given by the expert group is obtained. The method helps to judge the risk situation of knowledge sharing in a virtual enterprise and then contributes to the virtual enterprise's taking corresponding precautionary measures to evade the risks.

\section{Acknowledgment}

This work was partly supported by the Liaoning Provincial Science and Technology Department (Soft Science) Project of China (Project No. 76103059).

\section{References}

[1] W.X. Xu, Y.M. Wei and Y. Fan, "Virtual Enterprise and Its Intelligence Management”, Computers \& Industrial Engineering. 2002, 42: 199-205.

[2] M. Polanyi, “The Tacit Dimension,” London: Routledge and Kegan Paul, 1966.
[3] C.W. Holsapple, and M.P. Sena, “Organized Knowledge Sharing with Book-centered Web Sites: An Architecture, Implementation and Analysis," Information Technology and Management, 2000, (1): 363-377.

[4] S. Fernie, S.D. Green, S.J. Weller, and R. Newcombe, "Knowledge Sharing: Context, Confusion and Controversy," International Journal of Project Management, 2003, 21: 177187.

[5] P.F. Boone, "Managing Intra-corporate Knowledge Sharing,” Delft: Eburon, 1997.

[6] J. Liebowitz, and I. Megbolugbe, "A Set of Frameworks to Aid the Project Manager in Conceptualizing and Implementing Knowledge Management Initiatives,” International Journal of Project Management, 2003, 21: 189-198.

[7] W.M. McLure, and S. Faraj, "“It is what one does": why people participate and help others in electronic communities of practice?" Journal of Strategic Information System, 2000, 9(2-3): 155173.

[8] C. d'Aspremont, S. Bhattacharya, and L.A. Gérard-Varet. "Knowledge as a public good: efficient sharing and incentives for development effort,” Journal of Mathematic Economics, 1998, 30: 389-404.

[9] F. Ulrich. "The Knowledge-Based View (KBV) of the Virtual Web, the Virtual Corporation and the Net-Broker. Yogesh Malhotra, "Knowledge Management and Virtual Organizations”, Hershey USA and London UK: Idea Group Publishing, 2000.

[10] Rice, Majchrazak, King, Ba \& Malhotra. "Computer Mediated Interorganizational Knowledge Sharing: Insights form a Virtual Team Innovating Using a Collaborative Tool", Yogesh Malhotra, "Knowledge Management and Virtual Organizations", Hershey USA and London UK: Idea Group Publishing, 2000.

[11] Z.T. Zhou, "Research on Knowledge Sharing in Virtual Enterprise," Value Engineering, 2005, (9): 87-89 in Chinese.

[12] F. Herrera, E. Herrera-Viedma, and J.L. Verdegay, "Choice process for nonhomogeneous group decision making in linguistic setting”, Fuzzy Sets and Systems, 1998, 94(3): 287-308.

[13] F. Herrera, E. Herrera-Viedma, and J.L. Verdegay, "Direct approach processes in group decision making using linguistic OWA operators”, Fuzzy Sets and Systems, 1996, 79(1): 175-190.

[14] F. Herrera, and E. Herrera-Viedma, "Linguistic decision analysis: steps for solving decision problems under linguistic information”, Fuzzy Sets and Systems, 2000, 115(1): 67-82. 